\title{
Illness perceptions and coping determine quality of life in COPD patients
}

\author{
This article was published in the following Dove Press journal: \\ International Journal of COPD \\ 25 August 2016 \\ Number of times this article has been viewed
}

\section{Jitske Tiemensma' \\ Erin Gaab ${ }^{2}$ \\ Maarten Voorhaar ${ }^{3,4}$ \\ Guus Asijee ${ }^{3,5}$ \\ Adrian A Kaptein ${ }^{6}$}

'Psychological Sciences, ${ }^{2} \mathrm{Health}$ Sciences Research Institute, University of California, Merced, CA, USA; ${ }^{3}$ Department of Family Medicine, Maastricht University, Care and Public Health Research Institute School for Public Health and Primary Care, Maastricht, the Netherlands; ${ }^{4}$ Boehringer Ingelheim, Alkmaar, the Netherlands; ${ }^{5}$ Boehringer Ingelheim, Amsterdam, the Netherlands; ${ }^{6}$ Department of Medical Psychology, Leiden University Medical Centre, Leiden, the Netherlands
Correspondence: Jitske Tiemensma Psychological Sciences, University of California, 5200 North Lake Road, Merced, CA 95343, USA

Email jtiemensma@ucmerced.edu
Background: A key goal of chronic obstructive pulmonary disease (COPD) care is to improve patients' quality of life (QoL). For outcomes such as QoL, illness perceptions and coping are important determinants.

Aim: The primary aim was to assess the associations between illness perceptions, coping and QoL in COPD patients. A secondary aim was to compare illness perceptions and coping of patients with reference values derived from the literature.

Patients and methods: A total of 100 patients were included in the study. Patients were asked to complete the Brief Illness Perception Questionnaire (B-IPQ), the Utrecht Proactive Coping Competence scale (UPCC), and a QoL item. Correlations and linear regression models were used to analyze the data. Student's $t$-tests were used to compare patients with COPD with reference values derived from the literature.

Results: Patients with better understanding of COPD utilized more proactive coping strategies $(P=0.04)$. A more intense emotional response to COPD was related to less proactive coping $(P=0.02)$. Patients who reported using more proactive coping techniques also reported to have a better QoL $(P<0.01)$. Illness perceptions were also related to QoL: more positive illness perceptions were related to a better QoL (all $P<0.05$ ). Patients with COPD reported more negative illness perceptions than people with a common cold or patients with asthma (all $P<0.01$ ), but reported similar perceptions compared with patients with diabetes.

Conclusion: Patients with COPD reported a moderate QoL, but appeared to be proficient in proactive coping. Illness perceptions, coping, and QoL were all associated with each other. Patients reported more strongly affected illness perceptions compared to people with a cold and patients with asthma. We postulate that a self-management intervention targeting patients' illness perceptions leads to improved QoL.

Keywords: COPD, illness perceptions, coping, QoL, common sense model

\section{Introduction}

A key goal of chronic obstructive pulmonary disease (COPD) management is to increase patients' quality of life (QoL). When considering factors that influence outcomes in COPD care, perceptions about COPD and coping strategies are important determinants, ${ }^{1-5}$ especially in the context of self-management. The relation between illness perceptions, coping, QoL, and self-management is described in "The common sense model of self-regulation" (CSM). ${ }^{6}$ The CSM provides a framework for patients' perceptions, focusing on how emotions and thoughts about an illness are formed, coping strategies are chosen, and actions are taken. ${ }^{6}$ The CSM posits that individuals are active problem solvers who make sense of health threats by developing cognitive representations of the threats, which then influence strategies chosen to regulate the health threat within social and cultural contexts. Illness perceptions conceptualized in 
the CSM are persistent thoughts and feelings about a disease and/or its treatment. They impact how patients make sense of, and respond to, their illness. ${ }^{7}$ These subjective thoughts and emotions (ie, cognitions and affective responses) influence QoL and guide self-management. In the CSM, patients' perceptions are classified within the context of several components containing specific types of information about the threatening illness: ${ }^{7}$ the identity/label of the illness, the expected duration (chronic/acute and cyclical), cause of the illness, the expected consequences, overall comprehension of the illness, emotional responses, and the ability to control the illness (both on the personal level and the treatment level). ${ }^{8}$

Illness perceptions inform coping strategies. ${ }^{6}$ Coping has been defined as one's reaction to adverse events that require adjustment. ${ }^{9}$ It may involve behavioral and psychological responses and/or strategies to reduce stress. ${ }^{9}$ Coping strategies and styles have been described as passive or (pro)active, or as problem-focused or emotional. Proactive coping is defined as the process through which people detect or anticipate potential stressors and act in advance to mute the impact of the stressor or prevent the stressor altogether. ${ }^{10}$ Both illness perceptions and coping strategies are important determinants of QoL and self-management behaviors.

The present study primarily aims to assess the associations between illness perceptions, proactive coping and QoL in COPD patients. A secondary aim is to compare illness perceptions and proactive coping of patients with COPD with patients with other acute and chronic illnesses.

\section{Methods}

\section{Patients}

A total of 100 patients (58 males, 42 females) were included in the present study. Patients were selected from the pharmacy database based on their medication use. Patients who used COPD maintenance therapy for 1 year or longer were included in the study. On the demographics portion of the survey used in the present study, patients had to provide the date of COPD diagnosis. Inclusion criteria were age $>40$ years, being diagnosed with COPD at least 1 year ago, and using airway medication for at least a year. Patients were excluded if they were deemed unable to complete the questionnaires due to cognitive or linguistic impairments.

\section{Protocol}

Patients were selected through their pharmacist's database. Pharmacists randomly selected patients and contacted them by phone to inquire about their interest in participating in the study. Interested patients were invited to the pharmacy and handed an information leaflet and an informed consent form. After signing the consent form, patients were handed a packet of questionnaires and their medication data were extracted from the pharmacist's database. Questionnaires were returned by patients in a prestamped, addressed envelope. The study was approved by the Medical Ethics Committee of the Leiden University Medical Center (protocol number P11.170).

\section{Measures}

\section{Brief Illness Perception Questionnaire}

The Dutch language version ${ }^{11}$ of the Brief Illness Perception Questionnaire (B-IPQ) was used. The B-IPQ consists of eight single-item scales and part of a causal scale from the IPQrevised. ${ }^{8}$ The B-IPQ was designed to rapidly assess cognitive and emotional representations of illness. Every item is scored on a $0-10$ scale. Five of the items assess cognitive illness perceptions (eg, "How much do you think your treatment can help your illness?"), two items assess emotional perceptions (eg, “How does your illness affect you emotionally?"), and one item assesses illness comprehensibility (ie, "How well do you feel you understand your illness?"). The causal scale is an open-ended response item which asks participants to list the three most important self-perceived causal factors of their illness. ${ }^{12}$ The B-IPQ has been used in diverse age groups, illness types, and languages. A recent meta-analysis has shown that the scale has good concurrent validity and predictive validity. ${ }^{13}$ In the present study, the B-IPQ was shown to be reliable with a Cronbach's alpha of 0.702 .

\section{Utrecht Proactive Coping Competence scale}

The Utrecht Proactive Coping Competence scale (UPCC) ${ }^{14}$ consists of 21 items on a 1-4 Likert-type scale, ranging from not proficient to highly proficient. A total score (range 1-4) is calculated by averaging individual item scores. Higher scores on the UPCC indicate higher levels of perceived proactive coping. Examples of questions are "To what extent do you have the capacity to make realistic plans?" and "To what extent do you have the capacity to persist?" The UPCC has been found to be a reliable scale, which is positively associated with other measures of goal attainment, self-efficacy, planning, and health behaviors. ${ }^{14}$ The Cronbach's alpha was 0.934 in the present study. Furthermore, the scale has good construct validity and discriminant validity. ${ }^{14}$

\section{QoL}

QoL was assessed with a single question: "Could you indicate, generally speaking, how your quality of life is?" 
Patients were presented a $10 \mathrm{~cm}$ visual analog scale (VAS) ranging from 0 (bad) to 100 (good). A higher score on this item indicates a better QoL.

\section{Reference populations}

Because illness perceptions assess perceptions of a specific illness, there are no norm values for the general population. Therefore, reference groups from the literature were chosen to explore how patients with COPD are scoring compared with patients with other acute and chronic illnesses.

Reference values for the B-IPQ were extracted from the literature. Specifically, the values were obtained from the study that presented the brief version of the IPQ. ${ }^{12}$ The reference group with colds consisted of 49 subjects who were recruited from undergraduate classes at the University of Texas and who were asked to recall a recent illness $(n=49$, 21 males and 28 females, mean \pm SD age 18.0 \pm 0.5 years). This group was chosen because the common cold is an acute illness affecting the pulmonary system. The reference group of patients with asthma consisted of 309 patients who were recruited from general practitioner clinics in the UK using a postal survey $(n=319,127$ males and 182 females, mean \pm SD age $39.8 \pm 10.1$ years). This group was chosen as a comparison group because asthma shows similarity to COPD, in that both are obstructive lung diseases with breathlessness as a troublesome symptom. The last reference group consisted of patients with type-2 (T2) diabetes ( $\mathrm{n}=119$, who were recruited from outpatient clinics at Auckland Hospital, Auckland, New Zealand, 63 males and 56 females, mean \pm SD age $57.2 \pm 13.2$ years). This group was chosen based on T2 diabetes being a chronic illness like COPD.

To compare scores on the UPCC coping scale, reference groups were also obtained from the study that assessed the psychometric properties of the UPCC. ${ }^{14}$ The reference group of elderly (ie, above the age of 50 years) subjects participated in an intervention to prepare for aging $(n=158,52$ males and 106 females, mean \pm SD age $60.5 \pm 5.5$ years $).{ }^{15,16}$ The second reference group consisted of patients recently diagnosed with T2 diabetes $(\mathrm{n}=207,120$ males and 87 females, mean \pm SD age $62.3 \pm 5.4$ years $).{ }^{17,18}$

\section{Statistical analyses}

Data were analyzed using IBM SPSS Statistics version 23 (SPSS Inc., Chicago, IL, USA). Data are reported in tabular form, expressed as mean $\pm \mathrm{SD}$, unless stated otherwise. The primary analysis comprised the association between constructs of the CSM. To check the appropriateness of assumptions for the statistical analysis, Levene's test, histograms, and scatterplots were used. Bivariate correlations were calculated between illness perceptions, proactive coping, and QoL. Pearson's correlation coefficients were used, and Spearman's Rho was used in case of non-normally distributed data. Furthermore, two stepwise linear regression models were conducted (outcomes proactive coping and QoL). Age, sex, and severity of COPD were included as covariates in the regression models. The standardized Beta coefficients of these analyses were reported. There were no multicollinearity issues (all tolerance values $>0.40$ ). The level of significance was set at $P \leq 0.05$. The second analysis comprised of the comparison of results in patients with COPD and reference values from the literature. Mean values were calculated for all items of the B-IPQ and compared between groups using Student's $t$-test. The level of significance for this analysis was set at $P \leq 0.01$ because of multiple comparisons.

\section{Results \\ Sociodemographic and clinical characteristics}

The mean age of patients was 70 years ( $\mathrm{SD}=10$ years). Most patients were diagnosed with COPD over 3 years ago $(75 \%)$, with only 25 patients (25\%) being diagnosed between 1 and 3 years ago. The mean self-reported severity of COPD on a scale of 0-100 (with 100 being severe) was 39.27 ( $\mathrm{SD}=26.3$ ). Patients were prescribed a mean number of two medicines for COPD ( $\mathrm{SD}=0.9)$. All COPD related prescriptions are listed in Table 1

\section{Exploring associations between constructs of the CSM}

According to the CSM, illness perceptions inform coping strategies. Both illness perceptions and coping strategies can have an effect on QoL. Indeed, coherence was positively associated with proactive coping $(r=0.212, P=0.037)$, indicating that a better understanding of COPD is related to more proactive coping strategies. Likewise, emotional response was negatively associated with proactive coping ( $r=-0.240, P=0.018)$ which indicates that a more intense emotional response is related to less proactive coping.

As predicted by the CSM, proactive coping was positively associated with QoL ( $r=0.317, P=0.002)$. This association indicates that using more proactive coping strategies is related to better QoL.

Almost all dimensions of illness perceptions were related to QoL. Specifically, consequences were negatively correlated with QoL ( $r=-0.348, P=0.001)$, indicating that perceiving more consequences of COPD is associated with 
Table I Clinical characteristics

\begin{tabular}{|c|c|}
\hline Characteristics & COPD $(n=100)$ \\
\hline Sex (male/female) & $58 / 4 \mid$ ( $n=I$ unknown) \\
\hline Age (years) & $69.68 \pm 9.74$ \\
\hline \multirow[t]{4}{*}{ Educational level } & Low: 36 (36\%) \\
\hline & Medium: 39 (39\%) \\
\hline & High: 23 (23\%) \\
\hline & Unknown: 2 (2\%) \\
\hline \multirow[t]{3}{*}{ COPD diagnosis } & I-3 years ago: $20(20 \%)$ \\
\hline & More than 3 years ago: 75 (75\%) \\
\hline & Unsure: 5 (5\%) \\
\hline Self-reported COPD severity ${ }^{a}$ & $39.27 \pm 26.3$ \\
\hline \multirow[t]{10}{*}{ COPD medication } & Tiotropium: 97 (97\%) \\
\hline & Salmeterol: 42 (42\%) \\
\hline & Fluticasone: 20 (20\%) \\
\hline & Formoterol: I9 (19\%) \\
\hline & Budesonide: 9 (9\%) \\
\hline & Ciclesonide: 8 (8\%) \\
\hline & Ipratropium: 6 (6\%) \\
\hline & Indacaterol: 5 (5\%) \\
\hline & Theophylline: I (I\%) \\
\hline & Beclomethasone: I (I\%) \\
\hline Number of COPD medicines & $2.08 \pm 0.92$ \\
\hline Quality of life ${ }^{b}$ & $69.72 \pm 25.17$ \\
\hline UPCC total score ${ }^{c}$ & $2.97 \pm 0.45$ \\
\hline
\end{tabular}

Notes: Data are mean \pm SD or number and (\%); ${ }^{a}$ On a 0 (not severe) to 100 (severe) scale; ' $\mathrm{O}$ n a 0 (bad) to 100 (good) scale; ' On a I-4 scale (higher is better). Abbreviations: COPD, chronic obstructive pulmonary disease; UPCC, Utrecht Proactive Coping Competence scale.

a lower QoL. Likewise, timeline was negatively associated with QoL ( $r=-0.264, P=0.010)$, meaning that perceiving a more chronic timeline of COPD is related to lower QoL. Both personal control and treatment control were positively correlated with QoL ( $r=0.219, P=0.035$ and $r=0.223$, $P=0.029$, respectively). This means that higher perception of both personal control and treatment control is related to a better QoL. Identity was negatively associated with QoL ( $r=-0.502, P<0.001)$, indicating that perceiving more physical complaints due to COPD is related to a lower QoL. Concern was also negatively associated with QoL ( $r=-0.341$, $P=0.001$ ), which indicates that more concerns about COPD are related to a lower QoL. Lastly, emotional response was negatively correlated with QoL ( $r=-0.540, P<0.001)$, indicating that perceiving a more intense emotional response is related to a lower QoL. All significant associations are depicted in Table 2.

\section{The CSM}

Using a linear regression model, emotional response ( $\beta=-0.270, P=0.012)$ and illness coherence $(\beta=0.244$, $P=0.022)$ predicted proactive coping $\left(R^{2}=0.123\right)$, indicating that a lower emotional response and a better understanding of COPD predict proactive coping strategies. A second linear regression model revealed that QoL was predicted by proactive coping $(\beta=0.204, P=0.028)$ and emotional response $(\beta=-0.530, P<0.001)$. This model explained almost $38 \%$ of the variance $\left(R^{2}=0.377\right)$. The results indicate that more proactive coping and a less intense emotional response predict better QoL scores.

\section{Illness perceptions in patients with COPD compared with reference groups Illness perceptions in patients with COPD compared} with patients with the common cold

Compared with patients with the common cold, patients with COPD perceived more negative consequences $(P<0.001)$, a more chronic timeline $(P<0.001)$, and more physical complaints $(P=0.001)$, and were more concerned about their illness $(P<0.001)$. However, patients with COPD perceived more personal control $(P<0.001)$ and more treatment control $(P<0.001)$ than patients with a cold. The B-IPQ scores are listed in Table 3.

Table 2 Associations between illness perceptions, coping, and QoL

\begin{tabular}{lll}
\hline Scale & UPCC & QoL \\
\hline B-IPQ & & $-0.348^{* * *}(-0.513$ to -0.157$)$ \\
Consequences & & $-0.264^{* *}(-0.442$ to -0.066$)$ \\
Timeline & & $0.219 *(0.016$ to 0.404$)$ \\
Personal control & & $0.223 *(0.023$ to 0.405$)$ \\
Treatment control & & $-0.502^{* * *}(-0.638$ to -0.335$)$ \\
Identity & $0.212^{*}(0.013$ to 0.395$)$ & $-0.34 I^{* * *}(-0.508$ to -0.148$)$ \\
Concern & $-0.240^{*}(-0.420$ to -0.041$)$ & $-0.540^{* * *}(-0.668$ to -0.379$)$ \\
Coherence & & $0.317^{* *}(0.125$ to 0.485$)$ \\
Emotional response & & \\
UPCC & & \\
Total score & & \\
\hline
\end{tabular}

Notes: Correlation coefficients are displayed (Pearson's $R$ for normally distributed data, and Spearman's Rho for non-normally distributed data); $* P \leq 0.05$, $* * P \leq 0.0$, $* * * P \leq 0.00$ I. The $95 \%$ confidence intervals are given in the brackets.

Abbreviations: B-IPQ, Brief Illness Perception Questionnaire; QoL, quality of life; UPCC, Utrecht Proactive Coping Competence scale. 
Table 3 Comparison of B-IPQ scores between patients with COPD and other patient groups

\begin{tabular}{lllll}
\hline B-IPQ & $\begin{array}{l}\text { COPD } \\
(\mathbf{n}=100)\end{array}$ & $\begin{array}{l}\text { Cold }^{12} \\
(\mathbf{n}=\mathbf{4 9})\end{array}$ & $\begin{array}{l}\text { Asthma }^{12} \\
(\mathbf{n}=\mathbf{3 0 9})\end{array}$ & $\begin{array}{l}\text { T2 diabetes }^{12} \\
(\mathbf{n}=\mathbf{I 1 9})\end{array}$ \\
\hline Consequences & $\mathbf{5 . 0 3} \pm \mathbf{2 . 5}$ & $3.8 \pm 2.2^{* *}$ & $3.5 \pm 2.3^{* *}$ & $4.7 \pm 2.9$ \\
Timeline & $\mathbf{9 . 4 2} \pm \mathbf{I . 5}$ & $5.4 \pm 3 . I^{* *}$ & $8.8 \pm 2.2^{*}$ & $9.2 \pm 1.9$ \\
Personal control & $\mathbf{6 . 1 7} \pm \mathbf{2 . 3}$ & $4.7 \pm 2.5^{* *}$ & $6.7 \pm 2.4$ & $6.7 \pm 2.3$ \\
Treatment control & $\mathbf{6 . 9 5} \pm \mathbf{2 . 2}$ & $5.5 \pm 2.9^{* *}$ & $7.9 \pm 2.0^{* *}$ & $8.0 \pm 2.3^{* *}$ \\
Identity & $\mathbf{5 . 5 2} \pm \mathbf{2 . 4}$ & $4.5 \pm 2.4^{*}$ & $4.5 \pm 2.3^{* *}$ & $4.6 \pm 2.8^{*}$ \\
Concern & $\mathbf{5 . 0 3} \pm \mathbf{3 . 0}$ & $2.5 \pm 2.5^{* *}$ & $4.6 \pm 2.8$ & $7.0 \pm 3 . I^{* *}$ \\
Coherence & $\mathbf{7 . 5 4} \pm \mathbf{2 . 3}$ & $6.4 \pm 2.7^{* *}$ & $6.5 \pm 2.6^{* *}$ & $7.9 \pm 2.3$ \\
Emotional response & $\mathbf{4 . 4 3 \pm 2 . 9}$ & $3.8 \pm 2.9$ & $3.3 \pm 2.9^{* *}$ & $4.3 \pm 3.3$ \\
\hline
\end{tabular}

Notes: Data are mean $\pm S D, * P<0.0$, $* * P<0.001$ compared with patients with COPD. Bolded scores are scores of patients with COPD in the present study. Abbreviations: B-IPQ, Brief Illness Perception Questionnaire; COPD, chronic obstructive pulmonary disease.

Illness perceptions in patients with COPD compared with patients with asthma

Compared with patients with asthma, patients with COPD perceived more negative consequences $(P<0.001)$, a more chronic timeline $(P=0.002)$, and more physical complaints $(P<0.001)$. Patients with COPD perceived less treatment control $(P<0.001)$ and a more intense emotional response $(P<0.001)$ than patients with asthma. Patients with COPD did have a better understanding of their illness than patients with asthma $(P<0.001)$. The B-IPQ scores are listed in Table 3.

\section{Illness perceptions in patients with COPD compared} with patients with type 2 diabetes

Compared with patients with $\mathrm{T} 2$ diabetes, patients with $\mathrm{COPD}$ perceived less treatment control $(P<0.001)$ and more physical complaints $(P=0.01)$. However, patients with COPD were less concerned about their illness than patients with $\mathrm{T} 2$ diabetes $(P<0.001)$. The B-IPQ scores are listed in Table 3.

\section{Coping in patients with COPD compared with reference groups}

Compared with elderly subjects, patients with COPD scored significantly higher on the UPCC (total score $2.8 \pm 0.4$ versus $3.0 \pm 0.5$, respectively, $P=0.004$ ). This indicates that patients with COPD feel more proficient in using proactive coping strategies. There was no difference on the UPCC between patients with COPD and patients with $\mathrm{T} 2$ diabetes (total score $3.0 \pm 0.5$ versus $2.9 \pm 0.6$, respectively, $P>0.05$ ).

\section{Discussion}

In general, patients with COPD seemed to have a good understanding of their illness, were aware of the chronicity of COPD, and reported to use proactive coping strategies. However, their QoL was impaired. This finding of impaired QoL is in agreement with both Scharloo et al ${ }^{19}$ and Braido et al. ${ }^{20}$ In the present study, patients' illness perceptions were related to the coping strategy they used. Specifically, a better understanding of COPD resulted in more proactive coping strategies. A more intense emotional response to COPD was related to less proactive coping. Coping in turn, was positively related to QoL: patients who reported using more proactive coping techniques also reported to have a better QoL. Illness perceptions were also related to QoL and seemed to follow a similar pattern: more positive illness perceptions were related to a better QoL. These findings follow the structure of the CSM, which states that illness perceptions inform coping strategies. Furthermore, both illness perceptions and coping strategies can influence QoL. Our findings are in line with several studies, which all report similar patterns in patients with COPD: less positive illness perceptions in general, ${ }^{2,4,5}$ stronger illness identity, ${ }^{3,19,21-23}$ belief in a long illness duration, ${ }^{22,24}$ perceiving more severe consequences, ${ }^{3,22-24}$ and more intense emotional respons $\mathrm{e}^{3,19,22,23}$ were related to lower QoL. Interestingly, no other studies have observed an association between personal control and QoL in patients with COPD. We found that perceiving more personal control was related to better QoL. This finding is in agreement with multiple other studies in other illnesses (eg, acromegaly ${ }^{25}$ and Cushing's syndrome $\left.{ }^{26}\right)$. However, in the linear regression model only proactive coping and the emotional response were significant predictors for QoL. In addition to the relation between illness perceptions and QoL, Scharloo et a ${ }^{19}$ found that more passive coping was associated with worse QoL (specifically on the mental health domain). This is comparable to our finding of the positive association between proactive coping and QoL.

In addition to investigating the associations between the components of the CSM, we were also interested in illness perceptions and coping strategies as such. Illness perceptions, therefore, were compared to reference groups obtained from the literature. As expected, patients with COPD reported more negative illness perceptions than people with a common 
cold. Furthermore, patients with COPD reported more negative illness perceptions than patients with asthma, which we speculate, could be due to the more intense and chronic nature of COPD. Finally, patients with COPD reported similar illness perceptions when compared to patients with $\mathrm{T} 2$ diabetes. There were some differences; patients with COPD felt they had less treatment control and more physical symptoms, but they were less concerned about their illness than patients with T2 diabetes. On the UPCC coping scale, patients with COPD scored very similar to patients with diabetes and scored significantly better than elderly people.

The current study examined the relationship between QoL, illness perceptions, and coping following the CSM framework. A strength of the present study is the CSM construct comparison with reference populations from the literature. A limitation of the current study is the crosssectional nature, which prohibits exploring causal relationships. In addition, the sample size was relatively small. Future studies should incorporate a larger sample and a longitudinal design, in order to make causal statements about the relation between QoL, illness perceptions, and coping.

The results of the present study provide further evidence for the importance of self-management and disease-management interventions, which take illness perceptions and coping strategies into account. Rather than solely focusing on their pulmonary functions, patients should be encouraged to take control of their COPD and its treatment and consequences. ${ }^{27}$ Since a better understanding and less emotional responses are related to proactive coping and better QoL in patients with COPD, self-management interventions should target those perceptions. Encouraging proactive coping, helping patients to form realistic, optimistic views of the illness, and dispelling idiosyncratic beliefs may be paramount in improving QoL. Patients with COPD in the present study reported impaired QoL. The authors postulate that a self-management intervention targeting patients' (negative) illness perceptions might lead to improvements in QoL (see also Kaptein et al). ${ }^{28}$ As already suggested by numerous researchers, including Kaptein et al, ${ }^{27}$ the primary outcome in self-management should be patient-reported (ie, QoL) and not biomedical (eg, forced expiratory volume in 1 second).

\section{Conclusion}

In conclusion, patients with COPD reported affected illness perceptions and a moderate QoL, but appeared to be quite proficient in proactive coping. Illness perceptions, coping, and QoL were all associated with each other, as expected based on the CSM. Patients with COPD reported more negative illness perceptions than people with a common cold or patients with asthma. Patients with COPD felt they had less treatment control and more physical symptoms, but they were less concerned about their illness than patients with $\mathrm{T} 2$ diabetes. We postulate that a self-management intervention targeting patients' (negative) illness perceptions might lead to improvements in QoL.

\section{Disclosure}

GA and MV work at Boehringer Ingelheim, JT received a stipend from Boehringer Ingelheim for this study, and EG and AAK have no conflicts of interest in this work.

\section{References}

1. Kinsman RA, Yaroush RA, Fernandez E, Dirks JF, Schocket M, Fukuhara J. Symptoms and experiences in chronic bronchitis and emphysema. Chest. 1983;83(5):755-761.

2. Weldam SWM, Lammers J-WJ, Decates RL, Schuurmans MJ. Daily activities and health-related quality of life in patients with chronic obstructive pulmonary disease: psychological determinants: a crosssectional study. Health Qual Life Outcomes. 2013;11:190.

3. Weldam SWM, Lammers J-WJ, Heijmans MJWM, Schuurmans MJ. Perceived quality of life in chronic obstructive pulmonary disease patients: a cross-sectional study in primary care on the role of illness perceptions. BMC Fam Pract. 2014;15:140.

4. Vaske I, Kenn K, Keil DC, Rief W, Stenzel NM. Illness perceptions and coping with disease in chronic obstructive pulmonary disease: effects on health-related quality of life. J Health Psychol. February 2016. doi:10.1177/1359105316631197.

5. Mewes R, Rief W, Kenn K, Ried J, Stenzel N. Psychological predictors for health-related quality of life and disability in persons with chronic obstructive pulmonary disease (COPD). Psychol Health. 2016;31(4): 470-486.

6. Leventhal H, Meyer D, Nerenz D. The common sense representation of illness danger. In: Rachman S, ed. Contributions to Medical Psychology. New York, NY: Pergamon; 1980:7-30.

7. Leventhal H, Brissette I, Leventhal EA. The common-sense model of self-regulation of health and illness. In: Cameron LD, Leventhal H, eds. The Self-Regulation of Health and Illness Behaviour. London: Routledge; 2003:42-65.

8. Moss-Morris R, Weinman J, Petrie K, Horne R, Cameron L, Buick D. The Revised Illness Perception Questionnaire (IPQ-R). Psychol Health. 2002;17(1):1-16.

9. Aldwin CM, Brustrom J. Theories of coping with chronic stress. In: Gottlieb BH, ed. Coping with Chronic Stress. New York, NY: Plenum Press; 1997:75-103.

10. Aspinwall LG, Taylor SE. A stitch in time: self-regulation and proactive coping. Psychol Bull. 1997;121(3):417-436.

11. Kaptein AA, van Korlaar IM, Scharloo M. The Illness Perception Questionnaire. [webpage on the Internet] 2004. Available at: www. uib.no/ipq/html/b-ipq.html. Accessed July 24, 2015.

12. Broadbent E, Petrie KJ, Main J, Weinman J. The brief illness perception questionnaire. J Psychosom Res. 2006;60(6):631-637.

13. Broadbent E, Wilkes C, Koschwanez H, Weinman J, Norton S, Petrie KJ. A systematic review and meta-analysis of the Brief Illness Perception Questionnaire. Psychol Health. 2015;30(11):1361-1385.

14. Bode C, Thoolen B, de Ridder D. Het meten van proactieve copingvaardigheden. [Measuring proactive coping skills]. Psychol Gezondh. 2008;36(2):81-91. Dutch. 
15. Bode C, de Ridder DTD, Bensing JM. Preparing for aging: development, feasibility and preliminary results of an educational program for midlife and older based on proactive coping theory. Patient Educ Couns. 2006;61(2):272-278.

16. Bode C, de Ridder DTD, Kuijer RG, Bensing JM. Effects of an intervention promoting proactive coping competencies in middle and late adulthood. Gerontologist. 2007;47(1):42-51.

17. Thoolen B, De Ridder D, Bensing J, et al. Effectiveness of a selfmanagement intervention in patients with screen-detected type 2 diabetes. Diabetes Care. 2007;30(11):2832-2837.

18. Thoolen B, de Ridder D, Bensing J, Gorter K, Rutten G. Beyond good intentions: the development and evaluation of a proactive selfmanagement course for patients recently diagnosed with type 2 diabetes. Health Educ Res. 2008;23(1):53-61.

19. Scharloo M, Kaptein AA, Weinman JA, Willems LN, Rooijmans HG Physical and psychological correlates of functioning in patients with chronic obstructive pulmonary disease. J Asthma. 2000;37(1):17-29.

20. Braido F, Baiardini I, Balestracci S, et al. Chronic obstructive pulmonary disease patient well-being and its relationship with clinical and patientreported outcomes: a real-life observational study. Respiration. 2011; 82(4):335-340.

21. Scharloo M, Kaptein AA, Weinman J, et al. Illness perceptions, coping and functioning in patients with rheumatoid arthritis, chronic obstructive pulmonary disease and psoriasis. J Psychosom Res. 1998;44(5) $573-585$.
22. Scharloo M, Kaptein AA, Schlösser M, et al. Illness perceptions and quality of life in patients with chronic obstructive pulmonary disease. J Asthma. 2007;44(7):575-581.

23. Bonsaksen T, Haukeland-Parker S, Lerdal A, Fagermoen MS. A 1-year follow-up study exploring the associations between perception of illness and health-related quality of life in persons with chronic obstructive pulmonary disease. Int J Chron Obstruct Pulmon Dis. 2014;9:41-50.

24. Scharloo M, Kaptein AA, Weinman J, et al. Illness perceptions, coping and functioning in patients with rheumatoid arthritis, chronic obstructive pulmonary disease and psoriasis. J Psychosom Res. 1998;44(5): $573-585$.

25. Tiemensma J, Kaptein AA, Pereira AM, Smit JWA, Romijn JA, Biermasz NR. Affected illness perceptions and the association with impaired quality of life in patients with long-term remission of acromegaly. J Clin Endocrinol Metab. 2011;96(11):3550-3558.

26. Tiemensma J, Kaptein AA, Pereira AM, Smit JWA, Romijn JA, Biermasz NR. Negative illness perceptions are associated with impaired quality of life in patients after long-term remission of Cushing's syndrome. Eur J Endocrinol. 2011;165(4):527-535.

27. Kaptein AA, Fischer MJ, Scharloo M. Self-management in patients with COPD: theoretical context, content, outcomes, and integration into clinical care. Int J Chron Obstruct Pulmon Dis. 2014;9(1):907-917.

28. Kaptein AA, Scharloo M, Fischer MJ, et al. Illness perceptions and COPD: an emerging field for COPD patient management. J Asthma. 2008;45(8):625-629.
International Journal of COPD

\section{Publish your work in this journal}

The International Journal of COPD is an international, peer-reviewed journal of therapeutics and pharmacology focusing on concise rapid reporting of clinical studies and reviews in COPD. Special focus is given to the pathophysiological processes underlying the disease, intervention programs, patient focused education, and self management protocols.

\section{Dovepress}

This journal is indexed on PubMed Central, MedLine and CAS. The manuscript management system is completely online and includes a very quick and fair peer-review system, which is all easy to use. Visit http://www.dovepress.com/testimonials.php to read real quotes from published authors. 